\section{BONE}

\section{Function of soluble RANKL deciphered}

RANKL has key roles in the immune and skeletal systems, as well as in oncogenesis and metastasis. The cytokine occurs in two forms soluble and membrane-bound - and the precise role of the soluble form has so far been unclear. New research published in Nature Metabolism now

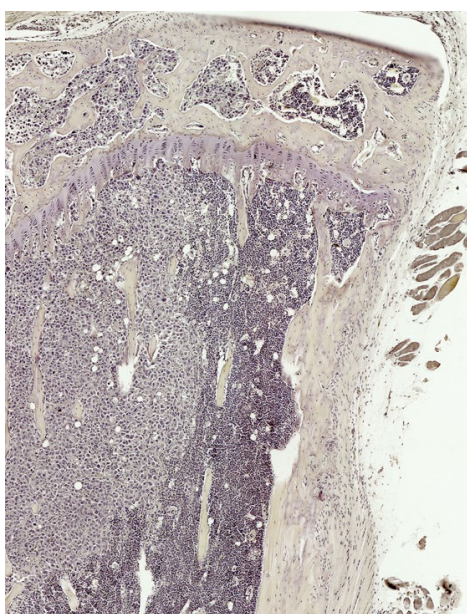

Bone metastasis of breast cancer cells in mice. Image courtesy of Kazuo Okamoto and Hiroshi Takayanagi, University of Tokyo, Japan. suggests that soluble RANKL is not required for physiological regulation of the skeletal and immune systems; however, soluble RANKL has an important role in the development of bone metastases.

"To clarify the role of soluble RANKL in vivo, we generated soluble RANKL-deficient mice by genetically deleting the cleavage sites of the extracellular domain of RANKL using the CRISPR-Cas9 system," explain authors Kazuo Okamoto and Hiroshi Takayanagi. Various analyses showed that these $\operatorname{Tn} f s f 11^{\Delta S / \Delta S}$ mice grew normally and had no obvious defects. They did not differ from control littermates in terms of growth, skeletal development, immune system function or bone remodelling, which demonstrates that soluble RANKL is not involved in these processes.

Next, the researchers injected wild-type and $\operatorname{Tn} f s f 11^{\Delta S / \Delta S}$ mice with B16F10 Red-FLuc cells (a melanoma cell line) to assess the role of soluble RANKL in tumour progression and metastases. Rapid metastasis into the long bones was seen in the wild-type mice. By contrast, tumour progression was reduced in the Tnfsf $11^{\Delta S / \Delta S}$ mice. Similar results were seen with the use of E0771-Luc cells (a breast cancer cell line). "Moreover, soluble RANKL deficiency affected neither osteoclasts at the metastasis site nor tumour metastasis to non-skeletal tissues," explain Okamoto and Takayanagi. "Thus, we showed that soluble RANKL is physiologically dispensable but promotes bone metastasis via directly triggering the migration of tumour cells to bone."

The researchers hope that their results will lead to the development of new therapies to specifically target the metastatic process. "We would like to establish strategies to block soluble RANKL alone without affecting membrane-bound RANKL or the production process of soluble RANKL in bone of patients with cancer," say Okamoto and Takayanagi.

Claire Greenhill

\section{of bone metastases}

ORIGINAL ARTICLE Asano, T. et al. Soluble RANKL is physiologically dispensable but accelerates tumour metastasis to bone. Nat. Metab. https:// doi.org/10.1038/s42255-019-0104-1 (2019)

\title{
THYROID DISEASE
}

\section{Long-term treatment outcomes for Graves disease}

Three therapies exist for Graves disease (also known as Graves hyperthyroidism): antithyroid drug therapy (ATD), radioactive iodine ( $\left(^{131}\right.$ therapy) to damage the thyroid or surgery to remove all or part of the thyroid. The efficacy of each of these treatments is well known; however, little is known about the long-term outcomes of each treatment modality. A new study in Thyroid presents long-term (6-10 years) outcome data for the different treatments of Graves disease.

The researchers recruited a cohort of 2,430 patients with newly diagnosed Graves disease from 13 endocrine clinics in Sweden in 2003-2005. Patients were followed up for $8 \pm 0.9$ years (mean \pm s.d.) after diagnosis. By reviewing the medical records of each patient and by conducting questionnaires, information was obtained on the treatment modality, patient demographic, lifestyle factors, rate of disease remission, quality of life and the presence of comorbidities.
The proportion of patients that were available at follow-up was $60 \%(1,186$ individuals). The first-line treatment of choice was ATD, followed by $\left.\right|^{131}$ therapy and then surgery. However, the remission rate after the first-line treatment was $45.3 \%$ (351/774) with ATD, 81.5\% (324/264) with ${ }^{131}$ therapy and $96.3 \%$ (52/54) with surgery.

Notably, if ATD-treated patients with disease recurrence underwent a second round of ATD, the rate of remission was even lower (29.4\%). Moreover, patients treated with ATD as a first-line therapy (with potential additional rounds of ATD) had a $49.7 \%$ risk of having either I $^{131}$ therapy or surgery (that is, ablative treatment) at follow-up. This situation is far from ideal, as it exposes patients to additional risks of adverse events from each treatment.

Of note, normal thyroid hormone status, without replacement with levothyroxine, was only achieved

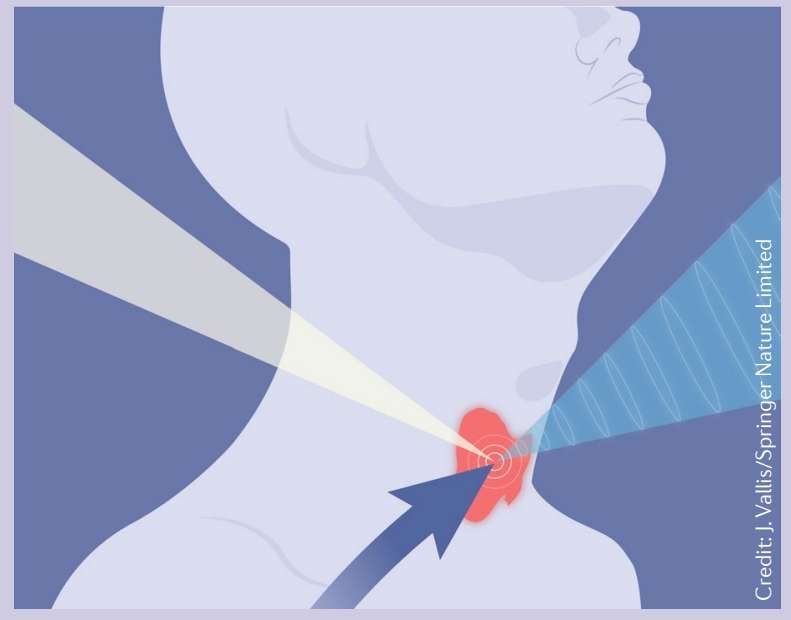

in $35.7 \%$ of all patient groups after follow-up. Moreover, $25.3 \%$ of patients did not feel fully recovered at follow-up.

The researchers conclude that patients who select ATD as a first-line treatment should be informed that they have only a $50.3 \%$ chance of avoiding ablative treatment and only a $40 \%$ chance of being euthyroid long term.

Shimona Starling

ORIGINAL ARTICLE Sjölin, G. et al. The long-term outcome of treatment for Graves' hyperthyroidism. Thyroid https://doi.org/10.1089/thy.2019.0085 (2019) 